continue post pregnancy. Pregnancy outcomes vary between Pakistani and white British pregnant women, but differences in health behaviours during pregnancy between these two groups are under researched.

Methods 4807 (1831 white British, 2222 Pakistani and 754 of Other origin) pregnant women were interviewed at 26-28 weeks of gestation using a questionnaire which collected information on alcohol, cigarette, caffeine, pregnancy vitamin and fruit and vegetable consumption and exercise levels. Latent class analyses were conducted to identify subgroups (classes) of the cohort defined according to clustering of health behaviours. The association between ethnicity, and other characteristics, with class membership was then examined.

Results Five independent classes of health behaviours were identified: three generally healthy classes that differed on alcohol and cigarette consumption, two unhealthy classes; one that smoked but didn't drink and one that was generally unhealthy. Although pregnant Pakistani and Other ethnicity women rarely reported smoking or alcohol consumption compared to white British women, other unhealthy behaviours such as lower rates of exercise and fruit and vegetable consumption were evident. Membership of the comprehensively unhealthy class was more likely in younger, white British pregnant women, of lower educational attainment.

Conclusions These techniques provide better understanding of negative behavioural clusters and characteristics associated with cluster membership. This could aid clinicians' ability to identify pregnant women who would benefit from interventions to modify these behaviours.

\section{P1-287 MATERNAL MORTALITY RATE IN KURDISTAN PROVINCE WESTERN IRAN FROM 2002 TO 2007; AN EPIDEMIOLOGIC SURVEY}

doi:10.1136/jech.2011.142976e.79

${ }^{1} \mathrm{~N}$ Esmailnasab, ${ }^{2} \mathrm{~A}$ Afkhamzadeh. ${ }^{1}$ Tabriz University of Medical Sciences, Tabriz, Iran; ${ }^{2}$ Kurdistan University of Medical Sciences, Sanandaj, Kurdistan, Iran

Introduction Nowadays 1500 mothers die due to complications of pregnancy and delivery in the world. This study is aimed to evaluation the frequency of maternal mortality rate and its associated causes in Kurdistan province Western Iran between 2002 and 2007.

Methods Data for this retrospective cross-sectional study was collected from the national surveillance system documents including information such as age, occupation, literacy, place of delivery, type of delivery, number of pregnancies, previous deliveries, operating labour, prenatal care, maternal mortality causes, and risk factors in labour.

Results A total of 46 maternal deaths were reported. Of them 22 cases $(47.8 \%)$ were lived in urban areas and 24 deaths $(52.2 \%)$ occurred in the rural areas. The most frequent maternal deaths were in the age group of $24-29$ years (39.2\%). Most died women were illiterate $(76.7 \%)$. The pick point of MMR occurred in the year 2004 (34.8\%). Most of deaths were occurred in hospitals (69.6\%). One fifth of operating labour were undertaken by uneducated midwifes. Overall, $27.9 \%$ of cases had not received any prenatal care during pregnancy or care was incomplete.

Conclusions Time trend of MMR during the period of study has significant changes, so that in the years 2002 to 2004 MMR in Kurdistan was higher than the national average. Poor prenatal care, low maternal education and health service shortages in rural areas in particular were the main risk factors associated with increased rate of MMR in western Iran.

\section{P1-288 PATTERNS OF SOCIAL INEQUALITY AMONG CASES OF MENINGOCOCCAL INFECTION IN SCOTLAND FROM 2005 TO 2008}

doi:10.1136/jech.2011.142976e.80

${ }^{1} \mathrm{C}$ Phillips, ${ }^{* 2} \mathrm{~J}$ Stevenson, ${ }^{2} \mathrm{G}$ MacKenzie. ${ }^{1}$ The University of Edinburgh, Edinburgh, UK ${ }^{2}$ NHS Lothian, Edinburgh, UK

Introduction Since the introduction of routine immunisation with the Meningococcal serogroup C vaccine (1999), the incidence of meningococcal infections, particularly Meningitis C infection, has steadily fallen in Scotland. However, despite the evident success of the vaccine there is still the issue that certain sub-groups of the Scottish population remain at disproportionate risk of acquiring the disease. We have explored the pattern of meningococcal cases in Scotland between 2005 and 2008 by socio-economic group.

Methods The Carstairs index, developed for 2001 census data, was used to match one of seven deprivation categories to the first four postcode digits of 548 Scottish meningococcal cases notified between 2005 and 2008.

Results Between 2005 and 2008 the incidence of meningococcal infection (per 100000) in Scotland showed a clear socio-economic gradient. While there was a clear socio-economic gradient in children (6.2 cases/100 000 for least deprived category, 12.6 cases/100 000 for most deprived category), there was no equivalent gradient observed for adults. There was no evidence of increased mortality in more deprived groups.

Conclusions There is a clear socio-economic gradient for meningococcal disease in children in Scotland. This finding is consistent with the internationally recognised influence of social inequality as a risk factor for worse health and increased susceptibility to infectious diseases, Despite major investment to reduce child poverty over the past decade it therefore appears that children from socially deprived areas have increased vulnerability to meningococcal infection. Explanations may include differences in housing, exposure to cigarette smoke and other social factors.

\section{P1-289 THE IMPORTANCE OF THE INVESTIGATION OF DEATHS AND THE COMMITTEE'S ACTION IN THE DIAGNOSIS OF MATERNAL MORBI-MORTALITY}

doi:10.1136/jech.2011.142976e.81

${ }^{2} \mathrm{M}$ C C Ferreira, ${ }^{1} \mathrm{R} P$ de Figueiredo, ${ }^{*} \mathrm{R}$ Laurenti, ${ }^{2} \mathrm{C}$ de Maria Barbosa Viana, ${ }^{2}$ A A A Falcão, ${ }^{2}$ C M C Ribeiro, ${ }^{2} \mathrm{~J}$ E V Câmara. ${ }^{1}$ Department of Epidemiology, School of Public Health, University of Sao Paulo, São Paulo, Brazil; ${ }^{2}$ Epidemiological Surveillance Division, Municipal Health Secretariat, João Pessoa-PB, Brazil

Introduction Reducing maternal mortality and guaranteeing universal access to reproductive health are related to the fifth Millennium Development goal; however, the identification of maternal deaths and the trustworthiness and comparability of the data are a challenge for epidemiological surveillance and the Maternal Mortality Committee (MMC), in assessing this information.

Methods All reproductive-aged women's deaths in the city of João Pessoa, Brazil, in 2005-2010, were investigated using multiple data sources (RAMOS). The MMC analysed this information for the correction of the Maternal Mortality Rate (MMR).

Results Investigation and data analysis showed that $44.4 \%$ of deaths (24360) of residents in João Pessoa during 2005-2010 were feminine. Of these, 1417 (13.1\%) involved women aged 10 to 49 years. Of the deaths investigated, 25 (1.8\%) occurred during pregnancy, childbirth or puerperium (PCP). The MMR found was $36.73 / 100000$ live-births, with no correction factor. The principal causes of maternal death were: puerperal infection (24\%) and 